OPEN ACCESS

Edited by:

Aristeidis H. Katsanos,

McMaster University, Canada

Reviewed by:

Christine Hawkes, McMaster University, Canada

Klearchos Psychogios,

Metropolitan Hospital, Greece

*Correspondence:

Albert J. YOO

ajyoo74@gmail.com

Specialty section:

This article was submitted to

Stroke,

a section of the journal

Frontiers in Neurology

Received: 19 February 2021 Accepted: 06 April 2021

Published: 11 May 2021

Citation:

Yoo AJ, Soomro J, Andersson T, Saver JL, Ribo M, Bozorgchami H, Dabus G, Liebeskind DS, Jadhav A,

Mattle $\mathrm{H}$ and Zaidat OO (2021) Benchmarking the Extent and Speed of Reperfusion: First Pass $\mathrm{TICl} 2 \mathrm{C}-3$ Is a Preferred Endovascular Reperfusion Endpoint. Front. Neurol. 12:669934. doi: 10.3389/fneur.2021.669934

\section{Benchmarking the Extent and Speed of Reperfusion: First Pass TICI 2c-3 Is a Preferred Endovascular Reperfusion Endpoint}

\author{
Albert J. Yoo ${ }^{1 *}$, Jazba Soomro ${ }^{1}$, Tommy Andersson ${ }^{2,3}$, Jeffrey L. Saver ${ }^{4}$, Marc Ribo ${ }^{5}$, \\ Hormozd Bozorgchami ${ }^{6}$, Guilherme Dabus ${ }^{7}$, David S. Liebeskind ${ }^{8}$, Ashutosh Jadhav ${ }^{9}$, \\ Heinrich Mattle ${ }^{10}$ and Osama O. Zaidat ${ }^{11}$ on behalf of ARISE II Investigators

\begin{abstract}
${ }^{1}$ Department of Neurointervention, Texas Stroke Institute, Fort Worth, TX, United States, ${ }^{2}$ Neuroradiology, Karolinska University Hospital, Clinical Neuroscience Karolinska Institutet, Stockholm, Sweden, ${ }^{3}$ Medical Imaging, Allgemeine Ziekenhuis Groeninge, Kortrijk, Belgium, ${ }^{4}$ Department of Neurology, University of California, Los Angeles, Los Angeles, CA, United States, ${ }^{5}$ Stroke Unit, Department of Neurology, Vall D'Hebron University Hospital, Barcelona, Spain, ${ }^{6}$ Department of Neurology, Oregon Health and Science University Hospital, Portland, OR, United States, ${ }^{7}$ Department of Interventional Neuroradiology, Miami Cardiac and Vascular Institute at Baptist Hospital of Miami, Miami, FL, United States, ${ }^{8}$ Department of Neurology, Neurovascular Imaging Research Core and Stroke Center, University of California, Los Angeles, Los Angeles, CA, United States, ${ }^{9}$ Department of Neurology, University of Pittsburgh Medical Center, Pittsburgh, PA, United States,

${ }^{10}$ Department of Neurology, Inselspital, University of Bern, Bern, Switzerland, ${ }^{11}$ Mercy St. Vincent Medical Center, Toledo, $\mathrm{OH}$, United States
\end{abstract}

Background and Purpose: End-of-procedure substantial reperfusion [modified Treatment in Cerebral Ischemia ( $\mathrm{mTICl}$ ) 2b-3], the leading endpoint for thrombectomy studies, has several limitations including a ceiling effect, with recent achieved rates of $\sim 90 \%$. We aimed to identify a more optimal definition of angiographic success along two dimensions: (1) the extent of tissue reperfusion, and (2) the speed of revascularization.

Methods: Core-lab adjudicated $\mathrm{TICl}$ scores for the first three passes of EmboTrap and the final all-procedures result were analyzed in the ARISE II multicenter study. The clinical impact of extent of reperfusion and speed of reperfusion (first-pass vs. later-pass) were evaluated. Clinical outcomes included 90-day functional independence [modified Rankin Scale (mRS) 0-2], 90-day freedom-from-disability (mRS 0-1), and dramatic early improvement [24-h National Institutes of Health Stroke Scale (NIHSS) improvement $\geq$ 8 points].

Results: Among 161 ARISE II subjects with ICA or MCA M1 occlusions, reperfusion results at procedure end showed substantial reperfusion in 149 (92.5\%), excellent reperfusion in 121 (75.2\%), and complete reperfusion in 79 (49.1\%). Reperfusion rates on first pass were substantial in 81 (50.3\%), excellent reperfusion in 62 (38.5\%), and complete reperfusion in 44 (27.3\%). First-pass excellent reperfusion (first-pass $\mathrm{TICl} 2 \mathrm{C}$ 3) had the greatest nominal predictive value for 90-day mRS 0-2 (sensitivity 58.5\%, specificity $68.6 \%$ ). There was a progressive worsening of outcomes with each additional pass required to achieve $\mathrm{TICl} 2 \mathrm{c}-3$. 
Conclusions: First-pass excellent reperfusion (TICl 2C-3), reflecting rapid achievement of extensive reperfusion, is the technical revascularization endpoint that best predicted functional independence in this international multicenter trial and is an attractive candidate for a lead angiographic endpoint for future trials.

Clinical Trial Registration: http://www.clinicaltrials.gov, identifier NCT02488915.

Keywords: intra-arterial therapy, reperfusion grading, reperfusion, brain ischaemia, cerebral infacrction, stent retriever, mechanical thrombectomy

\section{INTRODUCTION}

The current consensus statement-endorsed benchmark for procedural success after intra-arterial stroke therapy (IAT) is procedure end substantial reperfusion [modified Treatment in Cerebral Ischemia (mTICI) score of $2 \mathrm{~b}$ or higher], defined as the restoration of anterograde tissue perfusion in more than $50 \%$ of the target downstream territory $(1,2)$. The impressive clinical benefits observed in recent thrombectomy trials reflected improved reperfusion with second generation devices, most notably stent retrievers $(3,4)$. Since these pivotal trials, there has been a further increase in reported rates of substantial reperfusion (5). In the recent ARISE (Analysis of Revascularization in Ischemic Stroke with EmboTrap) II study, the core lab-adjudicated TICI $2 \mathrm{~b}-3$ rate at procedure end was 92.5\% (6).

However, there are considerable limitations to using the rate of TICI $2 b-3$ as a lead technical efficacy endpoint for IAT trials. First, this endpoint counts moderate reperfusion as a success, but when reperfusion is only $50-90 \%$ achieved, substantial tissue volumes remain in jeopardy. Second, the outcome of TICI $2 b-3$ is considered a success regardless of the number of passes required to achieve it, but maximal benefit is likely to be conferred by first-pass success, thus reducing ischemia duration (7). Third, the high rate of TICI $2 \mathrm{~b}-3$ seen with modern endovascular technology results in a ceiling effect, making the measure insensitive to further improvements in endovascular technique. Accordingly, a reevaluation of the optimal angiographic endpoint is necessary.

Using core-lab adjudicated data from ARISE II, we aimed to assess the clinical impact of the first-pass effect (FPE) and to identify the optimal definition of angiographic success along two dimensions: (1) the extent of tissue reperfusion, and (2) the speed of revascularization.

Abbreviations: ARISE II, Analysis of Revascularization in Ischemic Stroke with EmboTrap II study; ASPECTS, Alberta Stroke Program Early CT Score; ASTER, Direct Aspiration First Pass Technique for Thrombectomy Revascularisation of Large Vessel Occlusion in Acute Ischaemic Stroke; FP, first pass; FPE, first pass effect; HERMES, Highly Effective Reperfusion evaluated in Multiple Endovascular Stroke Trials Collaboration; IAT, intra-arterial stroke therapy; ICA, internal carotid artery; IQR, interquartile range; MCA, middle cerebral artery; mTICI, modified Treatment in Cerebral Ischemia; ROC, receiver-operating characteristic curve; sICH, symptomatic intracranial hemorrhage; TICI, Treatment in Cerebral Ischemia.

\section{MATERIALS AND METHODS}

All data generated or analyzed during this study are included in this published article and its Supplementary Material. The ARISE II study design and methods have been previously described (6). The study protocol was approved by the institutional review board/ethics committee at each participating site. All patients or their legally authorized representatives provided written informed consent before enrollment. To analyze a cohort with similar relationships between perfusion deficits and outcomes, only anterior circulation occlusions were included. To limit variability in the size of the at-risk territory, M2 occlusions were excluded, leaving only internal carotid artery (ICA) and middle cerebral artery (MCA) M1 occlusions in the study cohort. Angiographic endpoints were core lab-adjudicated and included TICI scores after each of the first three EmboTrap passes and final TICI score after all interventions. TICI scoring was inclusive of the $2 \mathrm{c}$ score (near complete or $>90 \%$ reperfusion of the downstream territory). Core lab readers were blinded to clinical outcomes.

\section{Statistical Analysis}

Baseline characteristics were reported using standard descriptive statistics. Parametric and non-parametric methods were applied where appropriate.

The clinical impact of first-pass reperfusion was measured controlling for final reperfusion grade. For example, among subjects with final TICI score of 3 , the subgroup where TICI 3 was achieved on the first pass was compared with the subgroup requiring multiple passes. These subgroups were compared on four efficacy and two safety outcomes. The primary efficacy outcome was 90-day functional independence [modified Rankin Scale (mRS) 0-2]. Additional efficacy outcomes were 90-day freedom from disability (mRS $0-1$ ), 90-day level of disability (ordinal 6-level mRS), and dramatic early improvement [24-h National Institutes of Health Stroke Scale (NIHSS) improvement $\geq 8$ points]. Safety outcomes were symptomatic intracranial hemorrhage (sICH) and 90-day mortality. Endpoints were assessed using the Chi-squared, Fisher's Exact, or Wilcoxon rank sum test as appropriate. This analysis was similarly done for subjects with final TICI $2 \mathrm{c}$ and separately $2 \mathrm{~b}$. Multiple logistic regression analysis for 90-day mRS 0-2 was performed to assess the impact of first-pass success adjusting for covariates with univariate $P<0.1$.

To explore the effect of final reperfusion extent on functional outcome, 90-day mRS scores were compared between final 
TICI grades using the Jonckheere-Terpstra trend test. Receiveroperating characteristic (ROC) analysis was used to identify the optimal TICI threshold for discriminating 90-day mRS 0-2 and $0-1$, and 24 -h NIHSS improvement of 8 or more points.

The extent of reperfusion at procedure end that was most strongly associated with outcomes was assessed by comparing: substantial reperfusion (mTICI 2b-3), excellent reperfusion (TICI 2c-3), and complete reperfusion (TICI 3). The extent measure that nominally performed best was then further tested to assess the impact of speed of attainment, by comparing achievement on first, second, third, or fourth or higher passes.

Because the core lab assessed reperfusion after each of the first three passes and at procedure end, all passes beyond the third were aggregated into a single category. The effect of the number of passes on 90-day mRS was evaluated using the JonckheereTerpstra trend test. ROC analysis was used to identify the optimal number of passes for predicting 90-day functional independence, 90-day freedom-from-disability and 24-h dramatic neurologic improvement. In all ROC analyses, the optimal operating point was defined as the point with the maximum Youden index $(=$ sensitivity + specificity -1$)$. Statistical significance was defined as two-tailed $P$-value $<0.05$.

Statistical analysis was performed using MedCalc Software version 19 (Ostend, Belgium). The conclusions were verified by an independent statistician using SAS version 9.4 software (Cary, NC).

ARISE II was sponsored by Neuravi, Inc., currently Cerenovus/Johnson \& Johnson. This study is the academic work of the authors. The sponsor played no role in the design and conduct of the study; collection, management, analysis, and interpretation of the data; preparation, review, or approval of the manuscript; and decision to submit the manuscript for publication.

\section{RESULTS}

\section{Overall Angiographic Results}

Two hundred twenty-seven patients were treated with EmboTrap in ARISE II. Nine basilar occlusion patients and 57 M2 occlusion patients were removed for this analysis. Baseline characteristics and outcomes are provided in Table 1. Of the 161 patients meeting study entry criteria, 149 (92.5\%) patients had substantial reperfusion at procedure end (final TICI 2b-3): 28 (17.4\%) final TICI 2b; 42 (26.1\%) final TICI 2c; and 79 (49.1\%) final TICI 3. The median number of thrombectomy passes was 2 [interquartile range (IQR) 1-3], and the highest number of passes was 9. Median procedural time was 44 (IQR 27-70) min.

Substantial reperfusion (TICI 2b-3) after the first pass was seen in $81(50.3 \%)$ patients: $19(11.8 \%)$ first-pass TICI $2 b ; 18$ (11.2\%) first-pass TICI 2c; and 44 (27.3\%) first-pass TICI 3. In the remainder, there were $53(32.9 \%)$ first-pass TICI $0-1$ and $27(16.8 \%)$ first-pass TICI 2a. Median procedural time from groin puncture to achieving TICI $\geq 2 \mathrm{~b}$ was 27 (IQR 22-38) min in the subjects who had first-pass TICI $2 \mathrm{~b}-3$ vs. 61 (IQR 46$86.5) \mathrm{min}$ in those who achieved TICI $2 \mathrm{~b}-3$ after two or more passes $(P<0.0001)$.
TABLE 1 | Baseline characteristics of study population $(n=161)$.

\begin{tabular}{lc}
\hline Variable & \\
\hline Age (years); mean \pm SD & $66.7 \pm 13.3$ \\
Female sex; $n$ (\%) & $92(57.1 \%)$ \\
Baseline NIHSS score; median (IQR) & $16(13-20)$ \\
Baseline NCCT ASPECTS; median (IQR) ( $n=124)$ & $10(10-10)$ \\
Occlusion level; $\boldsymbol{n}$ (\%) & \\
ICA & $35(21.7 \%)$ \\
MCA M1 & $126(78.3 \%)$ \\
IV tPA treatment; $n$ (\%) & $106(65.8 \%)$ \\
Hypertension; $n$ (\%) & $107(66.5 \%)$ \\
Diabetes mellitus; $n$ (\%) & $31(19.3 \%)$ \\
Atrial fibrillation; $n$ (\%) & $62(38.5 \%)$ \\
Dyslipidemia; $n$ (\%) & $63(39.1 \%)$ \\
Smoking; $n$ (\%) & $42(26.1 \%)$ \\
Previous stroke/transient ischemic attack; $n$ (\%) & $29(18.0 \%)$ \\
Previous MI/CAD; $n$ (\%) & $35(21.7 \%)$ \\
Clinical and safety outcomes & \\
24-h NIHSS score; median (IQR) ( $n=155)$ & $4(1-14)$ \\
Dramatic neurologic improvement [Baseline to 24-h & $94 / 155(60.6 \%)$ \\
NIHSS score improvement $\geq 8$ points; $n$ (\%)] & $1.5(0-4)$ \\
90-day disability level mRS; median (IQR) $(n=156)$ & $103 / 156(66.0 \%)$ \\
90-day functional independence, mRS 0-2; $n$ (\%) & $78 / 156(50.0 \%)$ \\
90-day freedom-from-disability, mRS 0-1; $n$ (\%) & $14 / 156(9.0 \%)$ \\
90-day mortality; $n$ (\%) & $10.2 \%)$ \\
sICH; $n$ (\%) & \\
\hline & \\
\hline
\end{tabular}

SD, standard deviation; NIHSS, National Institutes of Health Stroke Scale; IV TPA, intravenous tissue plasminogen activator; IQR, interquartile range; MI/CAD, myocardial infarction/coronary artery disease; mRS, modified Rankin Scale; NCCT ASPECTS, non-contrast CT Alberta Stroke Program Early CT Score; $\mathrm{SICH}$, symptomatic intracranial hemorrhage.

\section{First-Pass Success vs. Multiple Passes: Final Angiographic and Clinical Outcomes}

Supplementary Material enumerates the breakdown of final TICI results based on the first pass TICI score. When controlling for final reperfusion grade, there were significantly better 90day ordinal $\mathrm{mRS}$ outcomes among patients who achieved their final TICI score on the first pass compared to multiple passes (Figure 1). For final TICI 2b, the median 90-day mRS scores were 1 (IQR 0-3) vs. 3 (IQR 2-4) for the first-pass group vs. the multiple-pass group $(P=0.04)$. For final TICI $2 \mathrm{c}$, the median 90-day mRS scores were 0 (IQR 0-2) vs. 2 (IQR 1-4) (first-pass group vs. the multiple-pass group; $P=0.004)$. For final TICI 3 , the median 90-day mRS scores were 1 (IQR $0-2$ ) vs. 2 (IQR $1-5)$ (first-pass group vs. the multiple-pass group; $P=0.01$ ) There were no significant differences among the first-pass and multiple-pass groups for sICH and for 90-day mortality, although there were numerically more safety events in the multiple-pass groups in most cases (Table 2). After adjusting for age, baseline NIHSS score, baseline Alberta Stroke Program Early Computed Tomography Score (ASPECTS), vessel occlusion level, atrial fibrillation, and final TICI 2c-3 score, first-pass success (i.e., when the final TICI score is achieved on the first pass) was an 

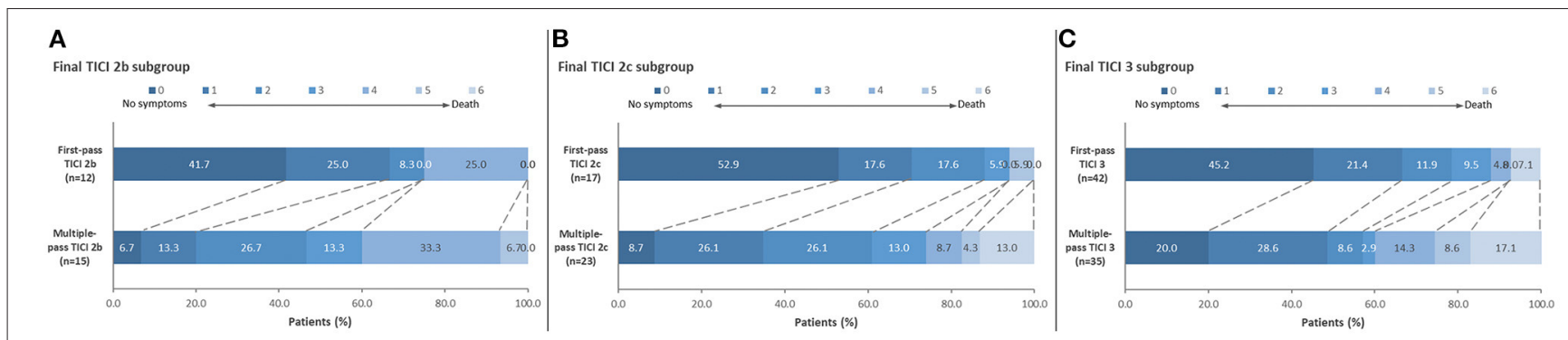

FIGURE 1 | Distribution of 90-day mRS scores comparing first-pass reperfusion vs. multiple-pass reperfusion. Results are shown for (A) final TICI 2b: median 90 -day mRS scores were 1 (IQR 0-3) vs. 3 (IQR 2-4) for the first-pass group vs. the multiple-pass group $(P=0.04)$; (B) final TICl 2c: median 90 -day mRS scores were 0 (IQR $0-2)$ vs. 2 (IQR 1-4) for the first-pass group vs. the multiple-pass group ( $P=0.004)$; and (C) final TICl 3 patients: median 90-day mRS scores were 1 (IQR 0-2) vs. 2 (IQR 1-5) for the first-pass group vs. the multiple-pass group $(P=0.01)$.

independent predictor of 90-day mRS 0-2 [odds ratio (OR) 3.42 (95\% CI, 1.27-9.17), $P=0.01]$. The associations between the baseline variables and both 90-day mRS 0-2 and final TICI 2c-3 are provided in Supplementary Material.

\section{Optimal Final TICI Score for Discriminating Good Clinical Outcome}

90-day functional outcome was significantly better with greater final reperfusion extent (Figure 2). Median 90-day mRS was 1 (IQR 0-3.25) for final TICI 3, 1.5 (IQR 0-3) for TICI 2c, 2 (IQR 1-4) for TICI $2 \mathrm{~b}$, and 3.5 (IQR 2-4.5) for TICI $0-2 \mathrm{a}(P$ $<0.05$; Jonckheere-Terpstra trend test). Final TICI 2c-3 showed the highest nominal accuracy for discriminating 90-day mRS 02 (sensitivity $79.6 \%$, specificity $34.0 \%$ ) and mRS 0-1 (sensitivity $83.3 \%$, specificity $33.3 \%$ ), and 24 -h NIHSS improvement $\geq 8$ points (sensitivity $80.9 \%$, specificity $34.4 \%$ ).

\section{Optimal Number of Passes for Achieving Final TICI 2c-3}

The median number of passes for patients with final TICI $2 c-3$ was one (IQR 1-3). Figure 3A illustrates the relationship between number of passes for achieving TICI $2 \mathrm{c}-3$ and 90 -day functional outcome. Median 90-day mRS was 1 (IQR 0-2) for one pass, 1 (IQR 1-4) for two passes, 2 (IQR 1-4) for three passes, and 2 (IQR 1-5) for four or more passes $(P=0.0001$; JonckheereTerpstra trend test). Median procedure time for one pass was 25 (IQR 20-34) min, for two passes was 44 (IQR 35-57.5) min, for three passes was 60 (IQR 48-64) $\mathrm{min}$, and for four or more passes was 76 (IQR 64.5-105) $\min (P<0.00001$; JonckheereTerpstra trend test). One pass showed the highest nominal accuracy for discriminating 90-day mRS 0-2 (sensitivity $58.5 \%$, specificity $68.6 \%$ ) and 24 -h NIHSS score improvement $\geq 8$ points (sensitivity $56.6 \%$, specificity $62.5 \%$ ). Importantly, there were no significant imbalances in the baseline variables between first-pass TICI 2c-3 and non-first-pass TICI 2c-3 (Table 3). Two or fewer passes was the optimal threshold for discriminating 90-day mRS 0-1 (sensitivity $80.0 \%$, specificity $46.2 \%$ ).

Regarding safety, each subsequent pass required to achieve TICI 2c-3 was associated with higher mortality: 90-day mortality was $5.1 \%(3 / 59)$ for one pass, $9.5 \%(2 / 21)$ for two passes, $16.7 \%$ $(3 / 18)$ for three passes, and $21.1 \%(4 / 19)$ for four or more passes
( $P=0.03$, Chi-squared trend test). For comparison, mortality in those without reperfusion (TICI 0-2a) was $16.7 \%$. There was no significant relationship between number of passes and $\mathrm{sICH}$. Rates of sICH were $1.6 \%$ (1/62) for one pass, $4.8 \%$ (1/21) for two passes, $10.5 \%$ (2/19) for three passes, and 5.3\% (1/19) for four or more passes $(P=0.20$, Chi-squared trend test). The sICH rate for TICI 0 -2a patients was $25 \%(3 / 12)$.

The impact of the number of passes to final TICI $2 c-3$ on 90-day outcome is shown separately for patients treated early from stroke onset $(\leq 4 \mathrm{~h}$ to groin puncture) vs. late $(>4 \mathrm{~h})$ in Supplementary Material.

\section{Likelihood of Achieving Final TICI 2c-3 Based on First-Pass Result}

There was a significantly higher chance of achieving TICI 2c-3 on the first pass $[38.5 \%(62 / 161)]$ compared to after the first pass [27.1\% (59/217); $P=0.02]$. Furthermore, if the first pass did not result in TICI $2 \mathrm{c}-3$ and further attempts were performed, there was a higher chance of achieving final TICI 2c-3 when the first pass yielded a lower reperfusion grade. Rates of final TICI $2 \mathrm{c}$ 3 were $71.7 \%$ (38/53) for first-pass TICI $0-1,55.6 \%(15 / 27)$ for first-pass TICI $2 \mathrm{a}$, and $50 \%(6 / 12)$ for first-pass TICI $2 \mathrm{~b}(P=$ 0.08; Chi-squared test for trend). There were more overall passes for lower first-pass TICI scores: median 3 (IQR 3-5) for firstpass TICI $0-1$ vs. 3 (IQR 2-3) for first-pass TICI 2a vs. 2 (IQR 2-2.5) for first-pass TICI $2 \mathrm{~b}(P=0.0004$; Jonckheere-Terpstra trend test).

\section{DISCUSSION}

Core-lab adjudicated ARISE II data confirm the superior clinical benefit of first-pass reperfusion. When adjusting for final TICI score, first-pass success (defined as when the final TICI score of $2 \mathrm{~b}-3$ is achieved on the first pass) yielded significantly better 90-day functional outcomes and was an independent predictor of 90-day independence. Furthermore, first-pass TICI 2c-3 was the optimal combination of reperfusion extent and speed for predicting good outcome after IAT. These findings were similar when restricting the analysis to subjects who received 3 or fewer passes with EmboTrap. 
The FPE has been variably defined as first-pass TICI 3 and more recently first-pass TICI $2 c-3(8-10)$. Our study supports the inclusion of $2 \mathrm{c}$ scores [defined in ARISE II as $>90 \%$ anterograde reperfusion (1)] into a standardized FPE definition. Final TICI $2 \mathrm{c}-3$ was achieved in $75.2 \%$ of the study cohort compared to $49.1 \%$ for TICI 3 alone and provided the best discrimination of good and excellent 90-day functional outcomes (mRS $0-1$ and $0-2$ ) and early dramatic neurologic improvement (24-h NIHSS improvement $\geq 8$ points). This mirrors previous reports that have found similar outcomes between TICI $2 \mathrm{c}$ and 3 reperfusion (11, 12). In an ancillary analysis of the ASTER trial, the magnitude of benefit for achieving 90-day mRS 0-2 was congruent between TICI $2 \mathrm{c}$ and 3 relative to TICI $2 \mathrm{~b}$, and combined TICI $2 \mathrm{c}-3$ patients had a significantly higher rate of favorable outcomes compared to $2 b$ patients [OR 1.72 (95\% CI, 1.01-2.90)] (12).

Another endpoint used in recent studies is first-pass TICI 2b-3, termed modified FPE $(8,9)$. A major limitation of this endpoint is that TICI $2 \mathrm{~b}$ encompasses an overly broad range of reperfusion results (50-89\% of the ischemic territory), many of which might be considered suboptimal currently. It is likely for this reason that the majority of first-pass TICI $2 b$ patients [12/19 (63\%)] underwent additional passes, questioning the clinical relevance of this first-pass category. There may be potential value of the expanded TICI (eTICI) scale, which subdivides TICI $2 \mathrm{~b}$ grades into eTICI $2 \mathrm{~b} 50$ (50-66\% reperfusion) and 2b67 (67$89 \%$ reperfusion). In the HERMES dataset, the c-statistic for discriminating 90-day mRS $0-2$ was slightly higher for eTICI (0.664) compared to the TICI classification employed in ARISE II (0.661), the only difference being the $2 \mathrm{~b} 67$ categorization (13). Future studies should investigate whether a reformulation of FPE to include eTICI $2 \mathrm{~b} 67$ results is warranted.

An additional argument against including TICI $2 \mathrm{~b}$ scores into the FPE definition is the issue of clot fragmentation, which can impede full reperfusion. Our analysis revealed a numerically lower likelihood of achieving TICI $2 \mathrm{c}-3$ when the first pass yielded TICI $2 \mathrm{~b}$ compared to a lesser score. Among patients who underwent multiple passes, there was a stepwise reduction in final TICI 2 c-3 rate from a first-pass TICI score of $0-1$ (72\%) to $2 \mathrm{a}$ $(56 \%)$ to $2 \mathrm{~b}(50 \%)(P=0.08)$. This observation likely owes to the smaller, more distal vessel segments that remain occluded when there is greater partial reperfusion, which are more difficult and riskier to treat. This increased risk may explain why there were fewer passes performed after first-pass TICI 2a and fewer still after TICI $2 \mathrm{~b}$ compared to TICI $0-1$. In this context, both TICI $2 \mathrm{a}$ and $2 \mathrm{~b}$ may be viewed as unwelcome indicators of thrombus fragmentation. Thrombus friability likely plays a central role in cases of distal embolization, and techniques should be targeted to minimize this phenomenon.

The optimal speed for attaining TICI $2 \mathrm{c}-3$ was one thrombectomy pass (first-pass TICI $2 \mathrm{c}-3$ ), which was observed in $51.2 \%$ of final TICI $2 c-3$ patients and best predicted 90 -day good outcome and early dramatic neurological improvement compared to other numbers of passes. There was a worsening of clinical outcomes with each additional pass, consistent with prior work showing that procedural time to reperfusion is a powerful predictor of IAT outcomes (7). With regards to benchmarking device performance, the number of passes is a 


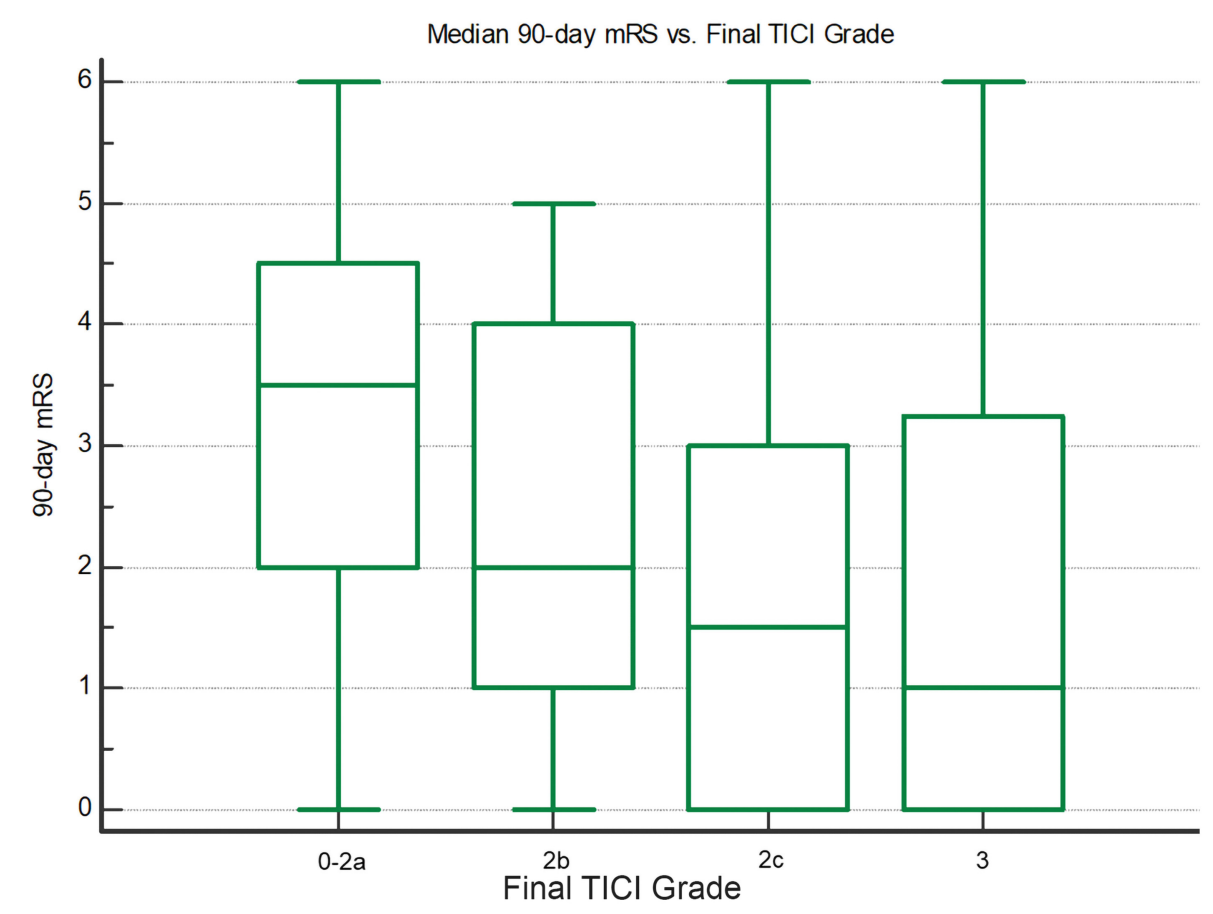

FIGURE 2 | Box-whisker plots showing median 90-day mRS vs. final TICI grade. There are significantly lower mRS scores with greater final reperfusion $(P<0.05$; Jonckheere-Terpstra trend test).

more suitable measure of device-related revascularization speed than procedure time because it disregards the time required for vessel catheterization, a delay which is unrelated to device action and can be highly variable (14).

A substantial proportion of study patients required three or more thrombectomy passes $(37 \%$ in the entire cohort, and $31 \%$ among those achieving final TICI 2c-3). In refractory cases, an important question facing neurointerventionists is how many passes should be performed before stopping. A recent study of stent retriever thrombectomy reported dismal outcomes after the third pass (7.4\% rate of 90-day mRS 0-2 for passes 4 through 8 ) despite an $\sim 25 \%$ per-pass rate of TICI $2 \mathrm{~b}-3$ (15). Conversely, another study of largely stent retrievers (95\%) found a significantly higher rate of good outcome in patients who achieved TICI $2 \mathrm{~b}-3$ on the fourth pass compared to nonreperfusers (16). Our analysis also suggests clinical value in pursuing more than three passes. First, even though the chance of achieving TICI $2 c-3$ dropped after the first pass, there was still a $30 \%$ per-pass rate of TICI $2 c-3$ among passes 3 or greater. And when TICI $2 c-3$ is achieved, there are higher rates of 90 -day mRS 0-2 and 0-1 (Figures 3A-C) for every pass number, including four or higher, compared to non-reperfusers (final TICI 02a). Although many of these per-pass comparisons were not statistically significant, this may be due to small sample sizes. The clinical value of near-complete/complete reperfusion achieved in a delayed fashion is consistent with other studies showing that complete reperfusion mitigates the deleterious effect of treatment delay $(16,17)$. In addition, there were no obvious safety concerns with pursuing more than three passes. Although mortality increased with each additional pass required to achieve TICI 2c-3, the mortality associated with 4 or more passes (21.1\%) was comparable to that seen in non-reperfusers (16.7\%). Concerning sICH, there was no significant association with pass number, and the rate of sICH with 4 or more passes $(5.3 \%)$ was lower than that seen in non-reperfusers (25\%). Previous reports are contradictory regarding the relationship between the number of passes and hemorrhagic conversion $(18,19)$.

Study limitations include use of a single device for the first three thrombectomy passes, which may limit generalizability. However, it is likely that the principal findings regarding the relationship between clinical outcome and the extent and speed of reperfusion are independent of how this reperfusion is achieved. Another limitation is that the study cohort comprised largely ideal treatment candidates, as reflected in their baseline ASPECTS scores (76\% with ASPECTS 10). As such, the outstanding outcomes reported here (66\% mRS 0-2 and $50 \%$ mRS $0-1$ at 90 days) do not reflect real-world practice. These limitations stem from the ARISE II study design as a prospective registration trial for FDA approval of EmboTrap. However, this design also lent numerous strengths to the analysis, including rigorous data collection and monitoring, minimal subject attrition (3\% at 90 days), and strict core lab adjudication of reperfusion results. Unlike previous thrombectomy studies, the ARISE II core lab prospectively evaluated each of the first 

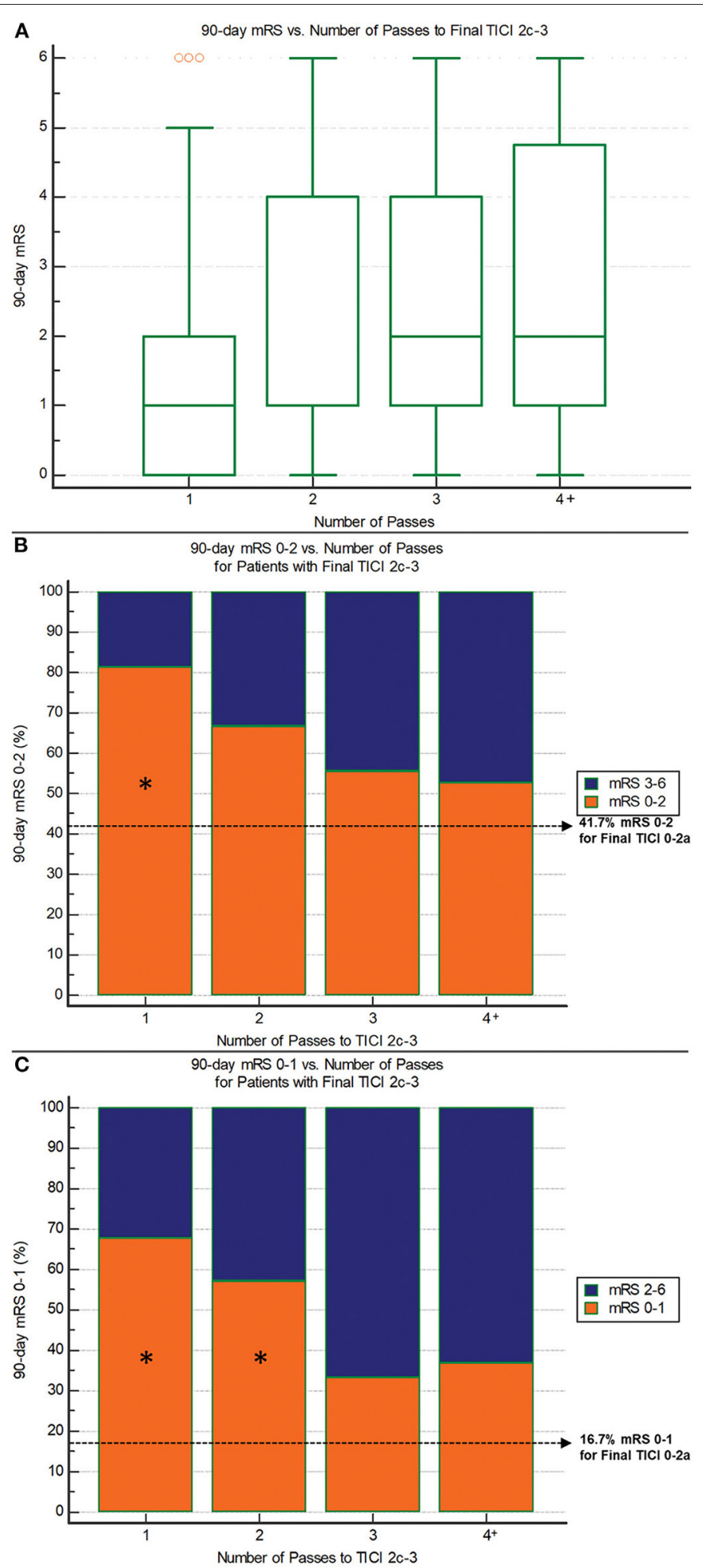

FIGURE 3 | 90-day outcomes vs. the number of passes required to reach final TICl 2c-3 reperfusion. (A) 90-day mRS: There are significantly lower mRS scores with fewer passes $(P=0.0001$; Jonckheere-Terpstra trend test). (B) 90-day mRS 0-2: There are progressively lower rates of good outcome with increasing number of passes $(P=0.005$; Chi-squared test for trend). Asterisk indicates significant difference compared to non-reperfusers (final TICl 0-2a). (C) 90-day mRS 0-1: There are progressively lower rates of excellent outcome with increasing number of passes $(P=0.003$; Chi-squared test for trend). Asterisk indicates significant difference compared to non-reperfusers (final $\mathrm{TICl} 0-2 \mathrm{a}$ ). 
TABLE 3 | Comparison of baseline variables between first-pass $\mathrm{TICl} 2 \mathrm{c}-3$ vs. non-first-pass TICl $2 \mathrm{c}-3$.

\begin{tabular}{|c|c|c|c|}
\hline Variable & FP TICI 2c-3 $(n=62)$ & Non-FP TICI 2c-3 $(n=59)$ & $P$-value \\
\hline Age (years); mean \pm SD & $67.2 \pm 12.8$ & $66.6 \pm 14.0$ & 0.78 \\
\hline Female sex; $n(\%)$ & $36(58.1 \%)$ & $36(61.0 \%)$ & 0.74 \\
\hline Baseline NIHSS score; median (IQR) & $16.5(12-19)$ & $17(14-21)$ & 0.36 \\
\hline Baseline NCCT ASPECTS; median (IQR) $(n=94)$ & $10(9.5-10)(n=48)$ & $10(9-10)(n=46)$ & 0.80 \\
\hline \multicolumn{4}{|l|}{ Occlusion level; $\boldsymbol{n}$ (\%) } \\
\hline ICA & $14(22.6 \%)$ & $13(22.0 \%)$ & 0.94 \\
\hline MCA M1 & $48(77.4 \%)$ & $46(78.0 \%)$ & \\
\hline IV tPA treatment; $n$ (\%) & $38(61.3 \%)$ & $44(74.6 \%)$ & 0.12 \\
\hline Hypertension; $n$ (\%) & 44 (71.0\%) & $40(67.8 \%)$ & 0.71 \\
\hline Diabetes mellitus; $n$ (\%) & $12(19.4 \%)$ & $11(18.6 \%)$ & 0.92 \\
\hline Atrial fibrillation; $n$ (\%) & $22(35.5 \%)$ & $25(42.4 \%)$ & 0.44 \\
\hline Dyslipidemia; $n$ (\%) & $22(35.5 \%)$ & $26(44.1 \%)$ & 0.34 \\
\hline Smoking; $n(\%)$ & $13(21.0 \%)$ & $18(30.5 \%)$ & 0.23 \\
\hline Previous stroke/transient ischemic attack; $n$ (\%) & $11(17.7 \%)$ & $9(15.3 \%)$ & 0.71 \\
\hline Previous MI/CAD; $n$ (\%) & $18(29.0 \%)$ & $11(18.6 \%)$ & 0.18 \\
\hline
\end{tabular}

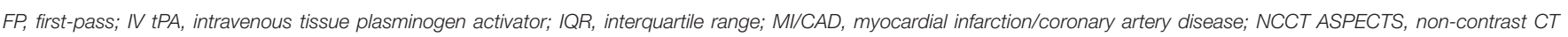
Alberta Stroke Program Early CT Score; NIHSS, National Institutes of Health Stroke Scale; SD, standard deviation; TICI, Treatment in Cerebral Ischemia scale.

three thrombectomy passes, yielding novel core lab-adjudicated data concerning reperfusion speed.

\section{CONCLUSIONS}

Data from ARISE II underscore the critical impact of procedural time to reperfusion on clinical outcomes after thrombectomy. First-pass TICI $2 c-3$ provides the optimal measure of both extent and speed of reperfusion for predicting good functional outcome and may serve as a useful benchmark for testing device performance and thrombectomy techniques in future studies.

\section{DATA AVAILABILITY STATEMENT}

The original contributions presented in the study are included in the article/Supplementary Material, further inquiries can be directed to the corresponding author/s.

\section{ETHICS STATEMENT}

The studies involving human participants were reviewed and approved by the institutional review board/ethics committee at each participating site. The patients/participants provided their written informed consent to participate in this study.

\section{REFERENCES}

1. Zaidat OO, Yoo AJ, Khatri P, Tomsick TA, von Kummer R, Saver $\mathrm{JL}$, et al. Recommendations on angiographic revascularization grading standards for acute ischemic stroke: a consensus statement. Stroke. (2013) 44:2650-63. doi: 10.1161/STROKEAHA.113.0 01972

2. Yoo AJ, Simonsen CZ, Prabhakaran S, Chaudhry ZA, Issa MA, Fugate JE, et al. Refining angiographic biomarkers of revascularization: improving

\section{AUTHOR CONTRIBUTIONS}

AY contributed to the conceptualization, methodology, software, formal analysis, and writing of the original draft of the manuscript. AY, TA, JSa, MR, HB, GD, DL, AJ, HM, OZ, and ARISE II Investigators participated in the investigation and provided resources and project administration. JSo contributed to the data visualization. AY, TA, JSa, MR, HB, HM, and $\mathrm{OZ}$ provided study supervision. AY, JSo, TA, JSa, $\mathrm{MR}, \mathrm{HB}, \mathrm{GD}, \mathrm{DL}, \mathrm{AJ}, \mathrm{HM}$, and $\mathrm{OZ}$ contributed to the review and editing of the final draft of the manuscript. All authors contributed to the article and approved the submitted version.

\section{ACKNOWLEDGMENTS}

Cerenovus sponsored the ARISE II study, and provided support for open access to this article.

\section{SUPPLEMENTARY MATERIAL}

The Supplementary Material for this article can be found online at: https://www.frontiersin.org/articles/10.3389/fneur. 2021.669934/full\#supplementary-material

outcome prediction after intra-arterial therapy. Stroke. (2013) 44:250912. doi: 10.1161/STROKEAHA.113.001990

3. Berkhemer OA, Fransen PS, Beumer D, van den Berg LA, Lingsma HF, Yoo AJ, et al. A randomized trial of intraarterial treatment for acute ischemic stroke. N Engl J Med. (2015) 372:11-20. doi: 10.1056/NEJMoa1411587

4. Goyal M, Menon BK, van Zwam WH, Dippel DW, Mitchell PJ, Demchuk AM, et al. Endovascular thrombectomy after large-vessel ischaemic stroke: a metaanalysis of individual patient data from five randomised trials. Lancet. (2016) 387:1723-31. doi: 10.1016/S0140-6736(16)00163-X 
5. Yoo AJ, Andersson T. Thrombectomy in acute ischemic stroke: challenges to procedural success. J Stroke. (2017) 19:121-30. doi: 10.5853/jos.2017.00752

6. Zaidat OO, Bozorgchami H, Ribo M, Saver JL, Mattle HP, Chapot R, et al. Primary results of the multicenter ARISE II study (analysis of revascularization in ischemic stroke with EmboTrap). Stroke. (2018) 49:110715. doi: 10.1161/STROKEAHA.117.020125

7. Alawieh A, Vargas J, Fargen KM, Langley EF, Starke RM, De Leacy R, et al. Impact of procedure time on outcomes of thrombectomy for stroke. J Am Coll Cardiol. (2019) 73:879-90. doi: 10.1016/j.jacc.2018.11.052

8. Zaidat OO, Castonguay AC, Linfante I, Gupta R, Martin CO, Holloway WE, et al. First Pass Effect: A New Measure for Stroke Thrombectomy Devices. Stroke. (2018) 49:660-6. doi: 10.1161/STROKEAHA.117.020315

9. Zaidat OO, Mueller-Kronast NH, Hassan AE, Haussen DC, Jadhav AP, Froehler MT, et al. Impact of balloon guide catheter use on clinical and angiographic outcomes in the STRATIS stroke thrombectomy registry. Stroke. (2019) 50:697-704. doi: 10.1161/STROKEAHA.118.021126

10. Nikoubashman O, Dekeyzer S, Riabikin A, Keulers A, Reich A, Mpotsaris A, et al. True first-pass effect. Stroke. (2019) 50:2140-46. doi: 10.1161/STROKEAHA.119.025148

11. Tung EL, McTaggart RA, Baird GL, Yaghi S, Hemendinger M, Dibiasio $\mathrm{EL}$, et al. Rethinking thrombolysis in cerebral infarction 2b: which thrombolysis in cerebral infarction scales best define near complete recanalization in the modern thrombectomy era? Stroke. (2017) 48:248893. doi: 10.1161/STROKEAHA.117.017182

12. Dargazanli C, Fahed R, Blanc R, Gory B, Labreuche J, Duhamel A, et al. Modified thrombolysis in cerebral infarction $2 \mathrm{C} /$ thrombolysis in cerebral infarction 3 reperfusion should be the aim of mechanical thrombectomy: insights from the ASTER trial (contact aspiration versus stent retriever for successful revascularization). Stroke. (2018) 49:1189-96. doi: 10.1161/STROKEAHA.118.0 20700

13. Liebeskind DS, Bracard S, Guillemin F, Jahan R, Jovin TG, Majoie CB, et al. eTICI reperfusion: defining success in endovascular stroke therapy. J Neurointerv Surg. (2019) 11:433-38. doi: 10.1136/neurintsurg-2018-0 14127

14. Ribo M, Flores A, Rubiera M, Pagola J, Mendonca N, Rodriguez-Luna D, et al. Difficult catheter access to the occluded vessel during endovascular treatment of acute ischemic stroke is associated with worse clinical outcome. $J$ Neurointerv Surg. (2013) 5 (Suppl. 1):i70-3. doi: 10.1136/neurintsurg-2012-0 10438

15. Flottmann F, Leischner $H$, Broocks $G$, Nawabi J, Bernhardt $M$, Faizy TD, et al. Recanalization rate per retrieval attempt in mechanical thrombectomy for acute ischemic stroke. Stroke. (2018) 49:2523-5. doi: 10.1161/STROKEAHA.118.022737
16. Garcia-Tornel A, Requena M, Rubiera M, Muchada M, Pagola J, Rodriguez-Luna D, et al. When to stop. Stroke. (2019) 50:1781-8. doi: 10.1161/STROKEAHA.119.025088

17. Prabhakaran S, Castonguay AC, Gupta R, Sun CJ, Martin CO, Holloway $\mathrm{W}$, et al. Complete reperfusion mitigates influence of treatment time on outcomes after acute stroke. J Neurointerv Surg. (2017) 9:3669. doi: 10.1136/neurintsurg-2016-012288

18. Bourcier R, Saleme S, Labreuche J, Mazighi M, Fahed R, Blanc R, et al. More than three passes of stent retriever is an independent predictor of parenchymal hematoma in acute ischemic stroke. J Neurointerv Surg. (2019) 11:625-9. doi: 10.1136/neurintsurg-2018-014380

19. Hassan AE, Kotta H, Shariff U, Preston L, Tekle W, Qureshi A. There is no association between the number of stent retriever passes and the incidence of hemorrhagic transformation for patients undergoing mechanical thrombectomy. Front Neurol. (2019) 10:818. doi: 10.3389/fneur.2019.00818

Conflict of Interest: AY is a consultant for Cerenovus, Penumbra, and Zoll, receives research grants from Medtronic, Cerenovus, Penumbra, Stryker, and Genentech, and has equity interest in Insera Therapeutics. TA is a consultant for Neuravi, Ablynx, Amnis Therapeutics, Medtronic, Rapid Medical, and Stryker. The University of California, Regents receives funding for JSa's services as a scientific consultant regarding trial design and conduct for Covidien and Stryker; JSa is an employee of the University of California, which holds a patent on retriever devices for stroke. MR is a shareholder in Anaconda Biomed, consultant for Cerenovus, Medtronic, Stryker, Apta Targets, and Vesalio. HB serves as a modest consultant for Cerenovus/Neuravi, and Stryker. GD serves as a consultant for Medtronic, Microvention, Penumbra, and Cerenovus. DL serves as an imaging core lab consultant for Cerenovus, Genentech, Medtronic, Stryker, and Vesalio. HM reports personal fees from Covidien/Medtronic, Neuravi/Cerenovus, Servier, and Bayer outside the submitted work, and served on the steering committees of the SWIFT PRIME and ARISE studies. OZ serves as a consultant for Neuravi, Stryker, Penumbra, and Medtronic.

The remaining authors declare that the research was conducted in the absence of any commercial or financial relationships that could be construed as a potential conflict of interest.

Copyright (c) 2021 Yoo, Soomro, Andersson, Saver, Ribo, Bozorgchami, Dabus, Liebeskind, Jadhav, Mattle and Zaidat. This is an open-access article distributed under the terms of the Creative Commons Attribution License (CC BY). The use, distribution or reproduction in other forums is permitted, provided the original author(s) and the copyright owner(s) are credited and that the original publication in this journal is cited, in accordance with accepted academic practice. No use, distribution or reproduction is permitted which does not comply with these terms. 